\title{
Standounkt
}

Soziales Tun im Kapitalismus

\section{Bewunderung trotz Zweifel}

\author{
Auch im alles bestimmenden Diktat der kapitalistischen \\ Gesellschaft blitzen immer wieder Menschen und soziale \\ Projekte auf, die sich diesem System wenn nicht widersetzen, \\ so doch dessen Handlungsmaximen reformulieren. Diese be- \\ wundernswerten Beispiele sind Gegenstand zahlreicher Bücher, \\ die neben dem Anregen der eigenen Reflexion motivieren \\ möchten, aktiv zu werden. Von Hans-Jürgen Arlt
}

$\mathbf{E}_{\mathrm{s}}^{\mathrm{s}}$ gibt kein richtiges Leben im falschen" - ist Theodor W. Adornos berühmtester Satz widerlegt? Sagt die gesellschaftskritische Behauptung, im Kapitalismus könne man nur leben, wenn man nicht so lebt, wie man leben möchte, mehr über unfähige Kritiker als über eine deformierte Gesellschaft? Der Boom der Bücher, die sich auf eine erfolgreiche Spurensuche selbstbestimmten, sinnerfüllten, sozial verantwortlichen Handelns begeben, wirft diese Frage auf.

Menschen machen die wunderbarsten Dinge, wenn sie und er ihre Zeit und Kraft nicht für sinnentleerte Erwerbsarbeit verausgaben, um das nötige und möglichst noch ein bisschen mehr Geld zu verdienen - diese Erfahrung berichten Autorinnen und Autoren aus allen Teilen dieser Welt. Die Meinungsführer der Arbeitsgesellschaft sagen etwas anderes. $\mathrm{Zu}$ viele Menschen seien Drückeberger, mit Vorliebe auf der Flucht vor den Anstrengungen der Arbeit in die Arme des Sozialstaates.

\section{Mehr als Arbeit oder Nichtarbeit}

Menschliche Aktivitäten streng nach Arbeit oder Nichtarbeit zu unterscheiden und die Arbeit primär nach wirtschaftlichen Kriterien zu organisieren, hat nichts Natürliches an sich, beides sind gesellschaftliche Prägungen. Für die Reinform wirtschaftlich organisierter Arbeit hat sich die Bezeichnung Kapitalismus eingebürgert. Je konsequenter und exklu- siver das Kriterium der Wirtschaftlichkeit durchschlägt, desto feingliedriger wird die Arbeitsteilung und desto dominanter das Geld. Hierzulande geht ohne Geld nichts mehr. Von der Schuhsohle bis zur Strähne im Haar, von der gekauften Wiege bis zur bezahlten Bahre erwerben wir die Arbeit anderer Menschen für cash oder auf Kredit.

Unser tägliches Tun gerät in einen Zangengriff. Auf der einen Seite wird die individuelle eigenständige Existenz an Gelderwerb und dieser für die meisten an Erwerbsarbeit gekoppelt. Auf der anderen Seite wird Erwerbsarbeit, siehe Taylorismus und Fordismus, möglichst restlos den Kriterien der Wirtschaftlichkeit unterworfen. Werden menschliche Aktivitäten in einen Handlungskorridor gedrängt, der Kosten zu minimieren und Erträge zu maximieren als Sperrgitter hat, dann sind es vorrangig drei Gesichtspunkte, unter welchen nach Alternativen Ausschau gehalten wird.

Erstens wird von einer individuellen Warte aus gefragt, ob es akzeptabel sein kann, seine Lebenszeit jenseits von Schlaf und Familie auf Tätigkeiten zu beschränken, deren primärer Sinn im Gelderwerb liegt. Daneben oder statt dessen „etwas Sinnvolles“ zu tun, halten viele für erstrebenswert. ,Daneben' nennt man es bürgerschaftliches Engagement; ,statt dessen' ist die Option der vielen, die eine bezahlte Arbeit in Non-Profit-Organisationen suchen. Wenn sie diese Option als Hochqualifizierte oder als Selbständige mit herausragendem Engagement umsetzen, haben sie die Chance als „Zukunftsmacher" oder als „Andersmacher“ vorgestellt zu werden.

Zweitens wird aus einer gesellschaftlichen Perspektive nach den negativen Folgen kapitalistischer Arbeit gefragt. Was bleibt unerledigt, weil es sich nicht rechnet, und vor allem, welche sozialen und ökologischen Schäden werden angerichtet? Wer sich der unerledigten Arbeit annimmt und etwas gegen die Schäden unternimmt, gilt als ,Social Entrepreneur. David Bornstein hat diesen Helden der Zivilgesellschaft ein ausgezeichnetes Buch gewidmet.

Drittens werden real existierende Unternehmer daraufhin beobachtet, ob unter ihnen nur pure oder auch „soziale Kapitalisten“ sind - Hannes Koch hat acht gefunden und porträtiert.

Jede dieser drei Reaktionen auf die kapitalistische Deformation der Arbeit hat ihre Berechtigung, jede verdient Respekt, weil sie sich dem normalen Gang der Dinge nicht anpasst. Die Belege dafür, dass es ein richtiges Leben im falschen gibt, sind beeindruckend. Sie schützen vor Verzweiflung, können aber die Zweifel nicht ausräumen, ob das Falsche dadurch richtiger wird.

\section{Literatur}

Bornstein, D.: Die Welt verändern. Social Entrepreneurs und die Kraft neuer Ideen. Stuttgart 2006 (2. Aufl.). 410 Seiten, 24,90 Euro.

Hildebrandt, A. / Howe, J. (Hrsg.): Die Andersmacher. Unternehmerische Verantwortung jenseits der Businessclass. Bielefeld 2008. 271 Seiten, 24,80 Euro.

Koch, H.: Soziale Kapitalisten. Vorbilder für eine gerechte Wirtschaft. Berlin 2007. 192 Seiten, 19,90 Euro.

Stefanska, J. / Hafenmayer, W.: Die Zukunftsmacher. Eine Reise zu Menschen, die die Welt verändern - und was Sie von ihnen lernen können. München 2007. 253 Seiten, 19,90 Euro.

AUTOR + KONTAKT

Dr. Hans-Jürgen Arlt arbeitet als Publizist und Kommunikationswissenschaftler in Berlin.

Dr. Hans-Jürgen Arlt, Perelsplatz 17, 12159 Berlin. Tel.: +493039501787, E-Mail: h-j.arlt@gmx.de, www.kommunikation-und-arbeit.de 
(c) 20I0 Authors; licensee IÖW and oekom verlag. This is an article distributed under the terms of the Creative Commons Attribution Non-Commercial No Derivates License (http://creativecommons.org/licenses/by-nc-nd/3.o/), which permits unrestricted use, distribution, and reproduction in any medium, provided the original work is properly cited. 